\title{
Doublet chemotherapy with cisplatin and pemetrexed is associated with a favorable outcome in patients with advanced non-squamous non-small-cell lung cancer who are eligible for bevacizumab and maintenance therapy
}

\author{
KAZUHISA NAKASHIMA ${ }^{1}$, HARUYASU MURAKAMI ${ }^{1}$, SHOTA OMORI $^{1}$, KAZUSHIGE WAKUDA $^{1}$, \\ AKIRA ONO $^{1}$, HIROTSUGU KENMOTSU ${ }^{1}$, TATEAKI NAITO ${ }^{1}$, MASAHIRO ENDO $^{2}$ and TOSHIAKI TAKAHASHI ${ }^{1}$ \\ Divisions of ${ }^{1}$ Thoracic Oncology and ${ }^{2}$ Diagnostic Radiology, Shizuoka Cancer Center, \\ Nagaizumi, Shizuoka 411-8777, Japan
}

Received March 1, 2016; Accepted July 29, 2016

DOI: $10.3892 / \mathrm{mco} .2016 .1001$

\begin{abstract}
The previous AVAPERL trial demonstrated that induction therapy with first-line cisplatin (CDDP), pemetrexed (PEM) and bevacizumab (BEV), followed by continuation maintenance therapy with PEM+BEV, improved the progression-free survival (PFS) and overall survival (OS) compared with BEV alone (median PFS, 10.2 vs. 6.6 months and median OS, 19.8 vs. 15.9 months, respectively) in patients with advanced non-squamous non-small-cell lung cancer (non-Sq NSCLC). However, those findings were based on selected patients who were eligible for $\mathrm{BEV}$ and maintenance therapy. To assess the efficacy of CDDP+PEM as first-line therapy in selected patients depending on their eligibility for BEV and maintenance therapy, consecutive patients with non-Sq NSCLC who received first-line chemotherapy with CDDP+PEM at the Shizuoka Cancer Center (Shizuoka, Japan) between July, 2009 and December, 2013 were retrospectively reviewed. A total of 160 patients were assessed, including 92 who were eligible and 68 who were not eligible for $\mathrm{BEV}$ treatment. In the BEV-eligible group, CDDP+PEM treatment followed by maintenance PEM exhibited significantly superior efficacy compared with that in the BEV-ineligible group (median PFS, 5.8 vs. 4.8 months, respectively, $\mathrm{P}=0.013$; and median OS, 21.3 vs. 12.6 months, respectively, $\mathrm{P}=0.0025$ ). In the BEV-eligible group, 60 patients were suitable for maintenance therapy with PEM (group A) and 32 patients were unsuitable (group B). In the BEV-ineligible group, 31 patients were suitable for maintenance therapy with PEM (group C) and 37 patients were unsuitable (group D). In group A, the median PFS and OS were 6.9 and 31.8 months, respectively, compared with 2.4 and
\end{abstract}

Correspondence to: Dr Kazuhisa Nakashima, Division of Thoracic Oncology, Shizuoka Cancer Center, 1007 Shimonagakubo, Nagaizumi-cho, Sunto-gun, Shizuoka 411-8777, Japan

E-mail:ka.nakashima@scchr.jp

Key words: non-squamous non-small-cell lung cancer, cisplatin, pemetrexed, bevacizumab, maintenance therapy
10.5 months in group B, 6.1 and 18.5 months in group C, and 2.8 and 7.7 months in group D. The PFS and OS in group A were significantly better compared with those in the other groups. Thus, the PFS and OS with CDDP+PEM were favorable among patients with advanced non-Sq NSCLC who were eligible for BEV and maintenance therapy.

\section{Introduction}

Lung cancer is the most common type of cancer worldwide, and is the leading cause of cancer-related mortality in men as well as in women. Approximately $85 \%$ of lung cancers are non-small-cell lung cancer (NSCLC), and $>70 \%$ of NSCLC patients present with inoperable and locally advanced (stage IIIB) or metastatic (stage IV) disease (1). Among patients with advanced NSCLC, the use of cytotoxic chemotherapy is associated with a response rate of $20-40 \%$ and a median survival time of 7-12 months (2-4). However, the prognosis of NSCLC patients has recently improved with the use of targeted therapies and new anticancer drugs.

Bevacizumab (BEV) is a monoclonal antibody targeted against vascular endothelial growth factor, and has been found to benefit patients with a variety of cancers. A phase III trial (E4599 study) established that the addition of BEV to first-line carboplatin + paclitaxel is an effective treatment for patients with advanced non-squamous (non-Sq) NSCLC (5). However, in the AVAiL study [BEV or placebo + cisplatin (CDDP) and gemcitabine], the progression-free survival (PFS) benefit did not translate into a significant overall survival (OS) benefit (6). Thus, the benefit of adding BEV to the CDDP regimen has not been demonstrated.

The randomized phase III AVAPERL study evaluated whether combination treatment with PEM and BEV in the maintenance setting could further improve the efficacy (compared with BEV monotherapy) in patients with advanced non-Sq NSCLC whose disease had not progressed after first-line induction treatment with CDDP, PEM and BEV $(7,8)$. The median PFS and OS with the induction treatment in the PEM+BEV arm were 10.2 and 


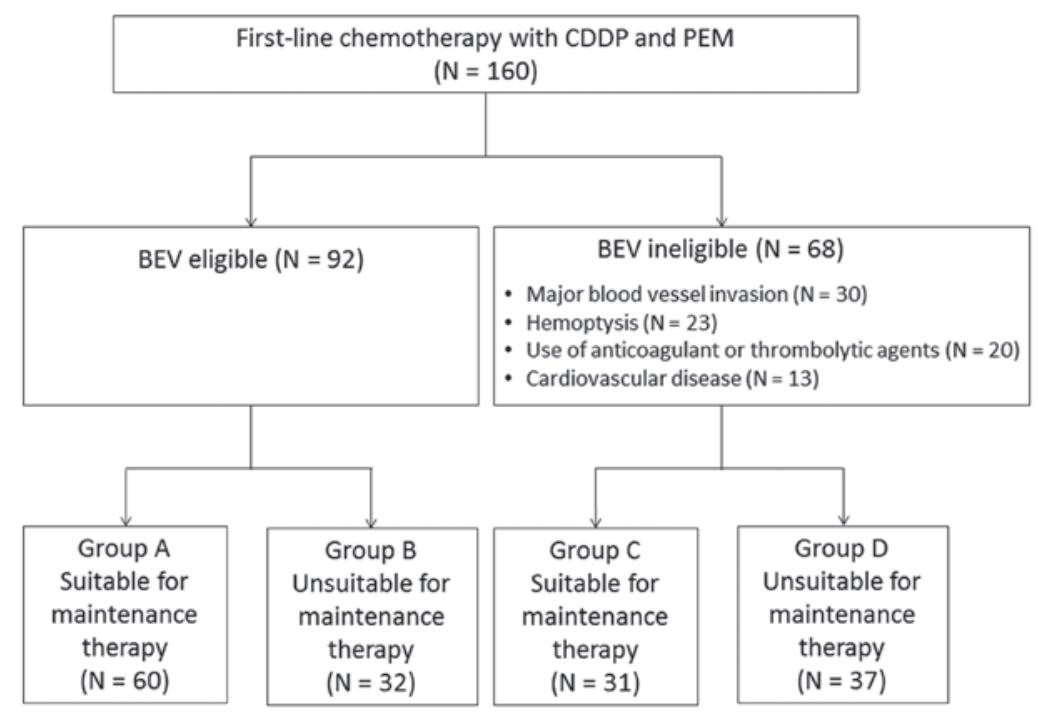

Figure 1. Flow diagram showing patient grouping in the present study. CDDP, cisplatin; PEM, pemetrexed; BEV, bevacizumab.

19.8 months, respectively, and these results were considered favorable for the treatment of advanced NSCLC. However, the AVAPERL study had several limitations, such as the survival data being based on selected patients who were eligible for BEV and maintenance therapy, and the lack of an arm for PEM alone as maintenance therapy. Therefore, the results of the AVAPERL study should be interpreted with extreme caution.

The present study retrospectively reviewed consecutive patients who received first-line chemotherapy with $\mathrm{CDDP}+\mathrm{PEM}$ in order to assess the efficacy of this regimen in selected patients who were eligible for BEV and maintenance therapy (similar to the AVAPERL study).

\section{Materials and methods}

Patients. Consecutive patients with stage IIIB/IV or recurrent non-Sq NSCLC who were initiated on first-line chemotherapy with CDDP $\left(75 \mathrm{mg} / \mathrm{m}^{2}\right)$ and PEM $\left(500 \mathrm{mg} / \mathrm{m}^{2}\right)$ between July, 2009 and January, 2013 at the Shizuoka Cancer Center (Shizuoka, Japan) were reviewed. The eligibility criteria for BEV according to the AVAPERL study were the absence of a history of hemoptysis, absence of evidence of a tumor invading the major vessels, absence of current or recent use of antithrombotic agents and absence of clinically significant cardiovascular disease. The eligibility criteria for maintenance therapy were the absence of disease progression and the absence of unacceptable toxicity at completion of four cycles of the induction therapy. The PFS and OS were then compared among patients who were eligible or ineligible for BEV therapy. Finally, the PFS and OS were compared among the maintenance therapy eligibility subgroups. The patient grouping process is summarized in Fig. 1.

The protocol of the present study was approved by the Institutional Review Board of the Shizuoka Cancer Center.

Statistical analysis. Differences in characteristics between the BEV-eligible and -ineligible groups were evaluated using Fisher's exact test. The PFS and OS were estimated using the Kaplan-Meier method, and the log-rank test was used for inter-group comparisons. The PFS and OS data were analyzed using JMP software, version 10.0 (SAS Institute, Cary, NC, USA).

\section{Results}

Patient characteristics. A total of 160 patients with stage IIIB/IV or recurrent non-Sq NSCLC who received first-line chemotherapy with CDDP and PEM were reviewed in the present study. A total of 92 patients were eligible for BEV therapy, whereas 68 patients were ineligible due to major blood vessel invasion $(n=30)$, hemoptysis $(n=23)$, use of anticoagulant or thrombolytic agents $(n=20)$ or cardiovascular disease $(n=13)$. The baseline characteristics, including gender, histology and stage, were similar between the BEV-eligible and -ineligible groups (Table I). However, compared with the BEV-ineligible group, the BEV-eligible group contained significantly more patients aged $<65$ years, with an Eastern Cooperative Oncology Group performance status of 0 , epidermal grow th factor receptor (EGFR) mutation-positive and never smokers.

Response to first-line CDDP+PEM in BEV-eligible vs. -ineligible non-Sq NSCLC patients. The overall response rate was $33 \%$ in the BEV-eligible and $35 \%$ in the BEV-ineligible group $(\mathrm{P}=0.74)$. However, treatment efficacy in the BEV-eligible group was significantly superior compared with that in the BEV-ineligible group in terms of survival (median PFS, 5.8 vs. 4.8 months, respectively, $\mathrm{P}=0.013$; and median $\mathrm{OS}$, 21.3 vs. 12.6 months, respectively, $\mathrm{P}=0.0025$ ) (Fig. 2).

Maintenance therapy with PEMs following first-line $C D D P+P E M$. The patients were stratified into groups A-D according to their eligibility for BEV and maintenance therapy (Fig. 1). In the BEV-eligible group, 60 patients were suitable for maintenance therapy (group A) whereas 32 were unsuitable (group B) due to adverse events or disease progression. In the $\mathrm{BEV}$-ineligible group, 31 patients were suitable for maintenance therapy (group C) and 37 patients were unsuitable (group D). In groups A and C, 22 (37\%) and 16 patients (52\%), respectively, received PEM maintenance therapy. The median PFS and OS 
Table I. Baseline patient characteristics.

\begin{tabular}{|c|c|c|c|}
\hline Characteristics & BEV-eligible (n=92) & BEV-ineligible $(n=68)$ & P-value \\
\hline \multicolumn{4}{|l|}{ Age (years) } \\
\hline Median (range) & $63(39-76)$ & $67(37-75)$ & 0.0036 \\
\hline$<65, \mathrm{n}(\%)$ & $61(66)$ & $29(43)$ & \\
\hline Gender, n (\%) & & & 0.085 \\
\hline Male & $58(63)$ & $52(76)$ & \\
\hline Female & $34(37)$ & $16(24)$ & \\
\hline Histology, n (\%) & & & 0.65 \\
\hline Adenocarcinoma & $90(98)$ & $65(96)$ & \\
\hline Other & $2(2)$ & $3(4)$ & \\
\hline Stage, n (\%) & & & 1 \\
\hline IIIB & $5(5)$ & $3(4)$ & \\
\hline IV & $87(95)$ & $65(96)$ & \\
\hline PS, n $(\%)$ & & & 0.004 \\
\hline 0 & $48(52)$ & $19(28)$ & \\
\hline 1 & $43(47)$ & $47(69)$ & \\
\hline 2 & $1(1)$ & $2(3)$ & \\
\hline EGFR mutation-positive, n (\%) & $22(24)$ & $7(10)$ & 0.037 \\
\hline Smoking status, n (\%) & & & 0.006 \\
\hline Current/former smoker & $60(65)$ & $58(85)$ & \\
\hline Never & $32(35)$ & $10(15)$ & \\
\hline
\end{tabular}

BEV, bevacizumab; PS, Eastern Cooperative Oncology Group performance status; EGFR, epidermal growth factor receptor.
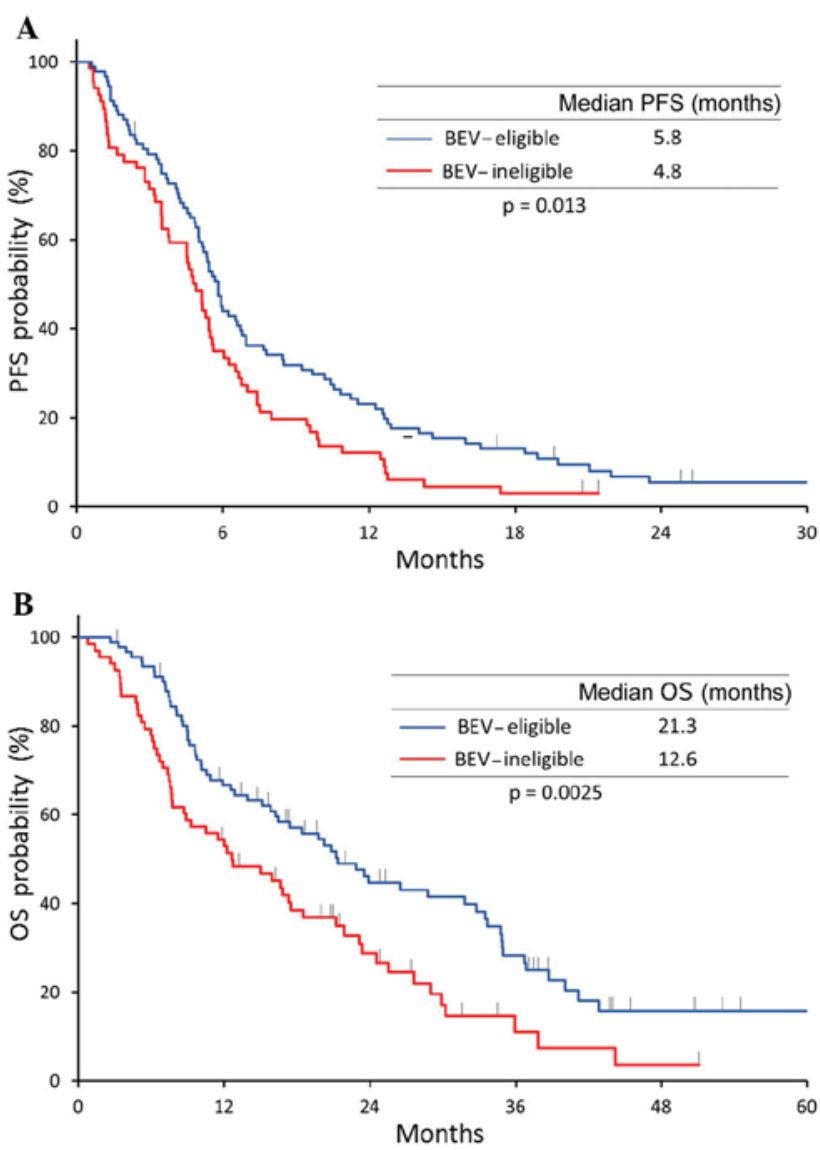

Figure 2. Kaplan-Meier curves for (A) progression-free survival (PFS) and (B) overall survival (OS) in patients treated with first-line cisplatin + pemetrexed stratified according to their eligibility for bevacizumab (BEV) treatment
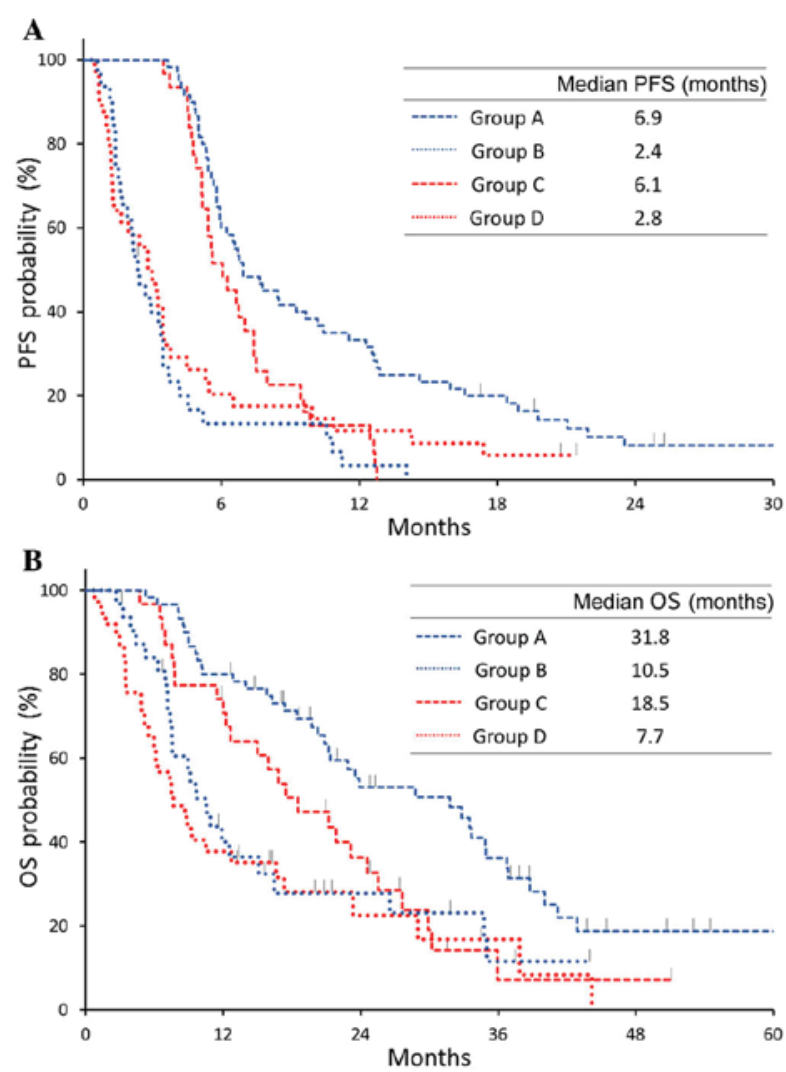

Figure 3. Kaplan-Meier curves for (A) progression-free survival (PFS) and (B) overall survival (OS) in patients treated with first-line cisplatin + pemetrexed stratified by eligibility for bevacizumab (BEV) and/or maintenance therapy (MT). Groups: A, eligible for both; B, eligible for BEV; C, eligible for MT; C, ineligible for both. 
were longer in groups $\mathrm{A}$ and $\mathrm{C}$ (maintenance therapy-eligible) compared with those in groups $\mathrm{B}$ and $\mathrm{D}$ (BEV-ineligible). In group A, the PFS and OS were 6.9 and 31.8 months, respectively, compared with 2.4 and 10.5 months in group B, 6.1 and 18.5 months in group $\mathrm{C}$, and 2.8 and 7.7 months in group D (Fig. 3). The PFS and OS in group A (eligible for both BEV and maintenance therapy) were significantly better compared with those in the other groups.

\section{Discussion}

In the present study, combination chemotherapy with CDDP and PEM achieved favorable PFS and OS in patients who were eligible for both BEV and maintenance therapy. This result supports the concept that eligibility for BEV represents a favorable prognostic factor, in accordance with the findings of a retrospective study by Takagi et al (9). In addition, patients who were eligible for maintenance therapy were found to have a better prognosis, which was expected, as patients with induction therapy failure were excluded. The favorable results of the AVAPERL study may have been affected by a similar selection bias. However, the AVAPERL study included a higher proportion of patients who were eligible for maintenance therapy (67\%) compared with the present study (57\%). Thus, the regimen used in the AVAPERL study (triplet induction chemotherapy with CDDP, PEM and BEV, followed by maintenance therapy with PEM and BEV) may be efficacious, although a similar OS was observed between the AVAPERL study and the BEV-ineligible group in the present study. In this context, although the addition of BEV improves tumor response, it may also lead to characteristic adverse events, such as hypertension, proteinuria, bleeding, hemoptysis and pulmonary embolism $(5,7,10)$. Furthermore, the AVAiL study failed to demonstrate an OS benefit with BEV therapy (6). The regimen used in the PARAMOUNT study (doublet induction therapy with CDDP and PEM, followed by continuation maintenance therapy with PEM), is a standard treatment for patients with advanced non-Sq NSCLC, based on the results of phase III trials $(11,12)$. However, whether the AVAPERL study regimen is superior to the PARAMOUNT study regimen remains unclear.

The present study has several limitations. First, the number of patients who were included in this retrospective study was relatively small. Furthermore, the subjects were Japanese patients, and their OS may be affected by subsequent therapy with EGFR tyrosine kinase inhibitors.

In conclusion, doublet induction chemotherapy with CDDP and PEM was associated with a favorable outcome in a patient population similar to that of the AVAPERL trial. Therefore, the PARAMOUNT study regimen is a reasonable treatment option for patients with advanced non-Sq NSCLC, regardless of their BEV eligibility status.

\section{Acknowledgements}

The authors would like to thank Editage (www.editage.jp) for the English language editing.

\section{References}

1. Molina JR, Yang P, Cassivi SD, Schild SE and Adjei AA: Non-small-cell lung cancer: Epidemiology, risk factors, treatment, and survivorship. Mayo Clin Proc 83: 584-594, 2008.

2. Azzoli CG, Baker S Jr, Temin S, Pao W, Aliff T, Brahmer J, Johnson DH, Laskin JL, Masters G, Milton D, et al: American Society of Clinical Oncology Clinical Practice Guideline update on chemotherapy for stage IV non-small-cell lung cancer. J Clin Oncol 27: 6251-6266, 2009.

3. Wakelee $\mathrm{H}$ and Belani CP: Optimizing first-line treatment options for patients with advanced NSCLC. Oncologist 10 (Suppl 3): S1-S10, 2005.

4. Sandler A: Bevacizumab in non small cell lung cancer. Clin Cancer Res 13 (Suppl): S4613-S4616, 2007.

5. Sandler A, Gray R, Perry MC, Brahmer J, Schiller JH, Dowlati A, Lilenbaum R and Johnson DH: Paclitaxel-carboplatin alone or with bevacizumab for non-small-cell lung cancer. $\mathrm{N}$ Engl J Med 355: 2542-2550, 2006.

6. Reck M, von Pawel J, Zatloukal P, Ramlau R, Gorbounova V, Hirsh V, Leighl N, Mezger J, Archer V, Moore N, et al: Overall survival with cisplatin-gemcitabine and bevacizumab or placebo as first-line therapy for nonsquamous non-small-cell lung cancer: Results from a randomised phase III trial (AVAiL). Ann Oncol 21: 1804-1809, 2010.

7. Barlesi F, Scherpereel A, Rittmeyer A, Pazzola A, Ferrer Tur N, Kim JH, Ahn MJ, Aerts JG, Gorbunova V, Vikström A, et al: Randomized phase III trial of maintenance bevacizumab with or without pemetrexed after first-line induction with bevacizumab, cisplatin, and pemetrexed in advanced nonsquamous non-small-cell lung cancer: AVAPERL (MO22089). J Clin Oncol 31: 3004-3011, 2013.

8. Baresi F, Scherpereel A, Gorbounova V, Gervais R, Vikström A, Chouaid C, Chella A, Kim JH, Ahn MJ, Reck M, et al: Maintenance bevacizumab-pemetrexed after first-line cisplatin-pemetrexed-bevacizumab for advanced nonsquamous nonsmall-cell lung cancer: Updated survival analysis of the AVAPERL (MO22089) randomized phase III trial. Ann Oncol 25: 1044-1052, 2014.

9. Takagi Y, Totiihara A, Nakahara Y, Yomota M, Okuma Y, Hosomi Y, Shibuya M and Okamura T: Eligibility for bevacizumab as an independent prognostic factor for patients with advanced non-squamous non-small cell lung cancer: A retrospective cohort study. PLoS One 8: e59700, 2013.

10. Reck M, von Pawel J, Zatloukal P, Ramlau R, Gorbounova V, Hirsh V, Leighl N, Mezger J, Archer V, Moore N and Manegold C: Phase III trial of cisplatin plus gemcitabine with either placebo or bevacizumab as first-line therapy for nonsquamous non-small-cell lung cancer: AVAiL. J Clin Oncol 27: 1227-1234, 2009.

11. Scagliotti GV, Parikh P, von Pawel J, et al: Phase III study comparing cisplatin plus gemcitabine with cisplatin plus pemetrexed in chemotherapy-naive patients with advanced-stage non-small-cell lung cancer. J Clin Oncol 26: 3543-3551, 2008.

12. Paz-Ares L, de Marinis F, Dediu M, Thomas M, Pujol JL, Bidoli P, Molinier O, Sahoo TP, Laack E, Reck M, et al: Maintenance therapy with pemetrexed plus best supportive care versus placebo plus best supportive care after induction therapy with pemetrexed plus cisplatin for advanced non-squamous non-small-cell lung cancer (PARAMOUNT): A double-blind, phase 3, randomised controlled trial. Lancet Oncol 13: 247-255, 2012. 